\title{
Optimized Sponge Layers, Optimized Schwarz Waveform Relaxation Algorithms for Convection-diffusion Problems and Best Approximation
}

\author{
Laurence Halpern ${ }^{1}$ \\ Laboratoire Analyse, Géométrie et Applications Universitè Paris XIII, 99 Avenue \\ J.-B. Clément, 93430 Villetaneuse, France. halpern@math.univ-paris13.fr \\ Summary. When solving an evolution equation in an unbounded domain, various \\ strategy have to be applied, aiming to reduce the number of unknowns and of com- \\ putation, from infinite to a finite but not too large number. Among them truncation \\ of domains with a sponge boundary and Schwarz Waveform Relaxation with over- \\ lap. These problems are closely related, as they both use the Dirichlet-to-Neumann \\ map as a starting point for transparent boundary condition on the one hand, and \\ optimal algorithms on the other hand. Then differential boundary conditions can \\ be obtained by minimization of the reflection coefficients-or the convergence rate. \\ In the case of unsteady convection diffusion problems, this leads to a non standard \\ complex best approximation problem that we present and solve.
}

\section{Problems settings}

\subsection{Absorbing boundary conditions with a sponge}

When computing for instance the flow passing an airfoil, or the diffraction by an object, the mathematical problem is set on an unbounded domain, while the domain of interest (i.e. where the knowledge of the solution is relevant), $\Omega_{I}$, is bounded and sometimes small . Then a computational domain is needed, called $\Omega_{C}$, on which the problem is actually solved. The problem must be complemented with boundary conditions on $\partial \Omega_{C}$. It is of importance to introduce a sponge boundary $\Omega_{S}$ which absorbs the spurious reflexion, see Figure 1 . The question we address here is the following : how to design boundary conditions on $\partial \Omega_{C}$ such that, for a given sponge layer of size $L$, the error in $\Omega_{I}$ be minimized. The issue is somewhat different from those used in the usual absorbing boundary condition setting, where there is no layer (see $[1,4,6]$ ), or in the classical sponge layer [7] or PML setting [2], where the equation is modified in the layer. 


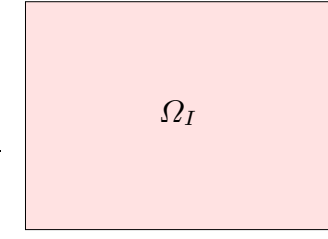

Domain of interest $\Omega_{I}$

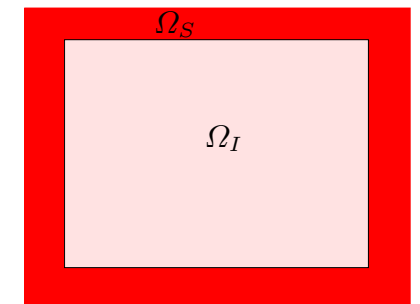

Domain of computation $\Omega_{C}=\Omega_{I} \cup \Omega_{S}$

Fig. 1. sponge boundary

\subsection{Domain decomposition with overlap}

Suppose now that the domain of interest $\Omega_{I}$ be too large to be treated by a single computer (like for instance in combustion problems, climate modelization, $\cdots)$. Then one can divide the domain into several parts, which overlap or not. In each domain the original problem is solved, whereas one has to supplement with transmission conditions between the subdomains. A model geometry is described in Figure 2.

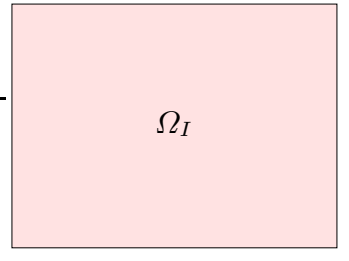

Domain of interest $\Omega_{I}$

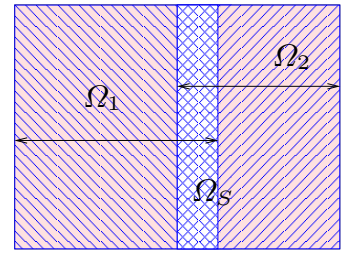

Splitted Domain

Fig. 2. Domain decomposition with overlap

In this case, given a size of the overlap, the transmission conditions are designed such to minimize the convergence rate of the Schwarz algorithm. The two procedures previously described lead to the same optimization problem, as we shall see in the next two sections. For the wave equation, an explicit answer was given in [5] for the low degrees. We present here the case of the unsteady reaction convection diffusion equation in $\mathbb{R}^{n+1}$

$$
\begin{gathered}
\mathcal{L}(u):=u_{t}-\nu \Delta u+a \partial_{x} u+\boldsymbol{b} \cdot \nabla u+c u=F \text { in } \mathbb{R}^{n+1} \times(0, T), \\
u(\cdot, 0)=u_{0} \text { in } \mathbb{R}^{n+1},
\end{gathered}
$$

where the coefficients are such that $\nu>0, a>0, \boldsymbol{b} \in \mathbb{R}^{n}, c>0$. The operator $\boldsymbol{\nabla}$ operates only in the $\boldsymbol{y}$ direction in $\mathbb{R}^{n}$. The more simple problem of designing absorbing boundary conditions, without sponge, has been addressed in [6], introducing an expansion in continous fractions. 
We first describe the methods in Sections 2 and 3, and we set the best approximation problem. In Section 4 we study this best approximation problem, which is defined in the complex plane, and involves a non linear functional. Therefore it is more involved than the standard one. In Section 5 we show numerical evidences for the optimality of the method.

\section{Sponge boundaries for the convection-diffusion equation: the half-space case}

A model problem is the following : the original domain is $\mathbb{R}^{n+1}$, the domain of interest is $\Omega_{I}=(-\infty, X) \times \mathbb{R}^{n}$ and the domain of computation is $\Omega_{C}=$ $(-\infty, X+L) \times \mathbb{R}^{n}$. A key point is that the data are compactly supported in $\Omega_{C}$.

\subsection{The transparent boundary condition}

As it is now classical, the transparent boundary condition on the boundary $\partial \Omega_{C}$ is obtained through a Fourier transform in time and in the transverse direction $\boldsymbol{y}$. Transforming the equation leads to

$$
-\nu \partial_{x x} \hat{u}+a \partial_{x} \hat{u}+\left(i(\omega+\boldsymbol{b} \cdot \boldsymbol{k})+\nu|k|^{2}+c\right) \hat{u}=0
$$

where $\hat{u}(x, \boldsymbol{k}, \omega)$ is the Fourier transform in the variables $\boldsymbol{y}$ and $t$. The characteristic equation is

$$
-\nu \lambda^{2}+a \lambda+i(\omega+\boldsymbol{b} \cdot \boldsymbol{k})+\nu|k|^{2}+c=0
$$

It has two roots, such that $\operatorname{Re} \lambda^{+} \geq a, \operatorname{Re} \lambda^{-} \leq 0$. The solution in the exterior of $\Omega_{C}$ can be written as

$$
\hat{u}(x)=\hat{u}(X+L) e^{\lambda^{-}(x-(X+L))}
$$

and the transparent boundary condition is given by

$$
\partial_{x} \hat{u}(X+L, \boldsymbol{k}, \omega)=\lambda^{-} \hat{u}(X+L, \boldsymbol{k}, \omega)
$$

We call $\Lambda^{-}$the pseudo-differential operator in the variables $\boldsymbol{y}$ and $t$ whose symbol is $\lambda^{-}$, and the original problem in $\mathbb{R}^{n+1}$ is equivalent to

$$
\begin{aligned}
& \mathcal{L}(u)=F \text { in } \Omega_{C} \times(0, T), \\
& u(\cdot, 0)=u_{0} \text { in } \Omega_{C}, \\
& \partial_{x} u(X+L, \boldsymbol{y}, t)=\Lambda^{-} u(X+L, \boldsymbol{y}, t)
\end{aligned}
$$




\subsection{Sponge boundaries : reflection coefficient}

Let now $v$ be a solution of problem with an approximate boundary condition

$$
\partial_{x} v(X+L, \boldsymbol{y}, t)=\Lambda_{a}^{-} v(X+L, \boldsymbol{y}, t),
$$

where $\Lambda_{a}^{-}$is an operator in the variables $\boldsymbol{y}$ and $t$, whose symbol $\lambda_{a}^{-}$will have to be a rational fraction in $\boldsymbol{k}$ and $\omega$. Ve introduce the reflection coefficient

$$
R\left(\omega, \boldsymbol{k}, \lambda_{a}^{-}\right)=\frac{\lambda^{-}-\lambda_{a}^{-}}{\lambda^{+}-\lambda_{a}^{-}} ; \quad \tilde{R}\left(\omega, \boldsymbol{k}, \lambda_{a}^{-}, L\right)=R\left(\omega, \boldsymbol{k}, \lambda_{a}^{-}\right) e^{-\lambda^{+} L}
$$

An easy calculation shows that the error between $u$ and $v$ is given by

$$
\|u-v\|_{L^{2}\left(\Omega_{I}\right)}^{2}=\int \frac{\left|\tilde{R}\left(\omega, \boldsymbol{k}, \lambda_{a}^{-}, L\right)\right|^{2}}{2 \operatorname{Re} \lambda^{+}}|\hat{u}(X, \omega, \boldsymbol{k})|^{2} d \omega d k
$$

In [6], it was proposed in the case $c=0$ to approximate $\lambda^{-}$by continuous fractions, for $L=0$, which produces a small error for small viscosity. For larger viscosities, another approach can be used, namely to search for $\lambda_{a}^{-}$in a class of rational fractions, which minimize the reflection coefficient. This will be done at the end of Section 3 .

\section{Overlapping Optimized Schwarz Waveform Relaxation methods for the convection-diffusion equation}

The model problem is the same as in Section 2. All the results in the next three sections can be found in [3]. The general Schwarz Waveform Relaxation algorithm for two domains $\Omega_{1}=(-\infty, L) \times \mathbb{R}^{n}, \Omega_{2}=(0, \infty) \times \mathbb{R}^{n}$ writes:

$$
\begin{gathered}
\left\{\begin{aligned}
\mathcal{L}\left(u_{1}^{k+1}\right) & =f & & \text { in } \Omega_{1} \times(0, T) \\
u_{1}^{k+1}(\cdot, 0) & =u_{0} & & \text { in } \Omega_{1} \\
\mathcal{B}_{1} u_{1}^{k+1}(L, \cdot) & =\mathcal{B}_{1} u_{2}^{k}(L, \cdot) & & \text { in }(0, T)
\end{aligned}\right. \\
\left\{\begin{aligned}
\mathcal{L}\left(u_{2}^{k+1}\right) & =f & & \text { in } \Omega_{2} \times(0, T) \\
u_{2}^{k+1}(\cdot, 0) & =u_{0} & & \text { in } \Omega_{2} \\
\mathcal{B}_{2} u_{2}^{k+1}(0, \cdot) & =\mathcal{B}_{2} u_{1}^{k}(0, \cdot) & & \text { in }(0, T)
\end{aligned}\right.
\end{gathered}
$$

A natural generalization of the Schwarz algorithm would be to use $\mathcal{B}_{1}$ and $\mathcal{B}_{2}$ as equal to identity. It can be proved to be convergent with overlap, with a convergence rate depending of the size of the overlap.

\subsection{The optimal Schwarz algorithm}

Theorem 1. The Schwarz method converges in two iterations with or without overlap when the operators $\mathcal{B}_{i}$ are given by:

$$
\mathcal{B}_{1}=\partial_{x}-\Lambda^{-}, \quad \mathcal{B}_{2}=\partial_{x}-\Lambda^{+},
$$

where $\Lambda^{ \pm}$are the operators whose symbols are the roots of (2). 


\subsection{The approximations by polynomials}

As in the case of absorbing boundary conditions, we choose approximate operators:

$$
\mathcal{B}_{1}^{a}=\partial_{x}-\Lambda_{a}^{-}, \quad \mathcal{B}_{2}^{a}=\partial_{x}-\Lambda_{a}^{+}
$$

Since $\Lambda^{-}$and $\Lambda^{+}$are related by $\Lambda^{-}+\Lambda^{+}=\frac{a}{\nu}$, we choose the approximations to be such that $\Lambda_{a}^{-}+\Lambda_{a}^{+}=\frac{a}{\nu}$. We define the error at step $k$ in domain $\Omega_{j}$ to be $e_{j}^{k}$. With the same notations as in previous section, and by analogous computations, we find the recursive relation

$$
\widehat{e_{j}^{k+2}}(\omega, 0, \boldsymbol{k})=\rho\left(\omega, k, \lambda_{a}^{-}, L\right) \widehat{e_{j}^{k}}(\omega, 0, \boldsymbol{k})
$$

where the CONVERGENCE RATE $\rho\left(\omega, \boldsymbol{k}, \lambda_{a}^{-}, L\right)$ is given by

$$
\rho\left(\omega, \boldsymbol{k}, \lambda_{a}^{-}, L\right)=R^{2}\left(\omega, \boldsymbol{k}, \lambda_{a}^{-}\right) e^{\left(\lambda^{-}-\lambda^{+}\right) L} .
$$

It measures the speed of convergence of the algorithm. The smaller it is, the faster the algorithm is. We rewrite it slightly differently. Let

$$
\delta(\omega, \boldsymbol{k})=a^{2}+4 \nu\left(i(\omega+\boldsymbol{b} \cdot \boldsymbol{k})+\nu|\boldsymbol{k}|^{2}+c\right) .
$$

We can write

$$
\lambda^{-}=\frac{a-\delta^{1 / 2}}{2 \nu}
$$

and $\delta^{1 / 2}(\omega, \boldsymbol{k})=f\left(i(\omega+\boldsymbol{b} \cdot \boldsymbol{k})+\nu|\boldsymbol{k}|^{2}\right)$ is approximated by a polynomial $P$ in the variable $i(\omega+\boldsymbol{b} \cdot \boldsymbol{k})+\nu|\boldsymbol{k}|^{2}$, and

$$
\lambda_{a}^{-}=\frac{a-P}{2 \nu} .
$$

Therefore the convergence rate takes the simple form

$$
\rho\left(\omega, \boldsymbol{k}, \lambda_{a}^{-}, L\right)=\left(\frac{P-\delta^{1 / 2}}{P+\delta^{1 / 2}}\right)^{2} e^{-2 \delta^{1 / 2} L / \nu} .
$$

In any case, in order to produce a convergent algorithm, we must have, $|\rho| \leq 1$ a.e. and $|\rho|<1$ on any compact set in $\mathbb{R} \times \mathbb{R}^{n}$. We notice that for a general polynomial $P$ we can have

$$
\lim _{(\omega,|\boldsymbol{k}|) \rightarrow+\infty}\left|\frac{P-\delta^{1 / 2}}{P+\delta^{1 / 2}}\right|=1 .
$$




\subsection{Approximate transmission conditions}

We consider here approximations of order lower than 1 . If $P=p+q x \in \mathbb{P}_{1}$, then

$$
\begin{aligned}
& \mathcal{B}_{1} \equiv \partial_{x}-\frac{a-p}{2 \nu}+q\left(\partial_{t}+\boldsymbol{b} \cdot \boldsymbol{\nabla}-\nu \Delta_{S}+c I\right), \\
& \mathcal{B}_{2} \equiv \partial_{x}-\frac{a+p}{2 \nu}-q\left(\partial_{t}+\boldsymbol{b} \cdot \boldsymbol{\nabla}-\nu \Delta_{S}+c I\right) .
\end{aligned}
$$

Theorem 2. For $p>0, q \geq 0, p>\frac{a^{2}}{4 \nu} q$, the algorithm is well-posed and converges with and without overlap.

The case $q=0$ corresponds to a polynomial of degree zero. This theorem is actually a composite of several results : first the algorithm is well-defined in relevant anisotropic Sobolev spaces : the result relies in trace theorems and energy estimates. Second the algorithms are convergent : in the non overlapping case, it relies again on energy estimates in each domain, arranged in such clever way as to cancelled out the terms on the boundary when suming up the estimates. In the overlapping case, the convergence rate is uniformly strictly bounded by one. The one-dimensional results can be found in [3], the twodimensional case without second order derivatives is treated in V. Martin's thesis and published in [8]. Her result extends to the case we present here without particular effort.

\section{The best approximation problems}

The convergence rate has two parts: the overlap intervenes in the term

$e^{-2 \delta^{1 / 2} L}$. Thus, in presence of an overlap, high frequency are taken care of by the overlap. In any case, when numerical schemes are involved, only discrete frequencies are present, and they are bounded from below and above. Let $Y_{j}$ be the maximum size of the domain in the $y_{j}$ direction/ If $\delta t$ and $\left\{\delta y_{1}, \cdots, \delta y_{n}\right\}$ are the discrete steps in time and space, the frequencies can be only such that $\omega \in I_{T}, k_{j} \in I_{j}$, with $I_{T}=\left(\frac{\pi}{T}, \frac{\pi}{\delta t}\right)$, and $I_{j}=\left(\frac{\pi}{Y_{j}}, \frac{\pi}{\delta y_{j}}\right)$. The best approximation problem consists in, for a given $n$, finding $P$ in $P_{n}$ minimizing $\sup _{\omega \in I_{T}, k_{j} \in I_{j}}\left|\rho\left(\omega, k, \lambda_{a}^{-}, L\right)\right|$.

Using the forms in (4) and (5), we can rewrite it as, for a given $n$, finding $P$ in $P_{n}$ minimizing

$$
\sup _{z \in K}\left|\frac{P(z)-f(z)}{P(z)+f(z)} e^{-L f(z) / \nu}\right|
$$

where $K$ is a compact set in $\mathbb{C}+, K=\left\{i(\omega+\boldsymbol{b} \cdot \boldsymbol{k})+\nu|\boldsymbol{k}|^{2}, \omega \in I_{T}, k_{j} \in\right.$ $\left.I_{j}, 1 \leq j \leq n\right\}$.

\subsection{A general result}

$K$ is a compact set in $\mathbb{C}, f$ a continuous function on $K$, such that $f(K) \subset$ $\{z \in \mathbb{C}: \operatorname{Re} z>0\}$. Define 


$$
\delta_{n}(l)=\inf _{p \in \mathbb{P}_{n}} \sup _{z \in K}\left|\frac{p(z)-f(z)}{p(z)+f(z)} e^{-l f(z)}\right|,
$$

Problem (6) generalizes as:

$$
\text { Find } p_{n}^{*} \text { such that } \sup _{z \in K}\left|\frac{p_{n}^{*}(z)-f(z)}{p_{n}^{*}(z)+f(z)} e^{-l f(z)}\right|=\delta_{n}(l)
$$

This is a non classical complex best approximation problem, for two reasons: first the cost function $\frac{p(z)-f(z)}{p(z)+f(z)}$ is non linear, second there is a weight $e^{-l f(z)}$ which is rapidly small and allows for large values of $\frac{p(z)-f(z)}{p(z)+f(z)}$. We have a fairly complete theory in the non overlapping case : existence, uniqueness, and equioscillation property. Furthermore any local minimum is global. In the overlapping case, general results are more restrictive:for $l$ sufficiently small, there is a solution, any solution equioscillates, and if $\delta_{n}(l) e^{l \sup _{z \in K} \Re f(z)}<1$, then the solution is unique. In the symmetric case, i.e., if $K$ is symmetric with respect to the real axis, and if for any $z$ in $K, f(\bar{z})=\overline{f(z)}$, then the polynomial of best approximation has real coefficients. Furthermore for odd $n$ the number of equioscillations is bigger or equal to $n+3$.

\subsection{The 1-D case}

In this case, the convergence rate actually equioscillates in 3 real points, and we can have explicit formulae to determine the best polynomial $p_{1}^{*}$. Furthermore the constrains on the coefficients for well-posedness are fulfilled. In 2-D, it is still an open question. When solving by a numerical scheme, the overlap is such that $L \approx C_{1} \Delta x$ and the space and time meshes are related by $\Delta t \approx C_{2} \Delta x^{\beta}, \beta \geq 1$ (in general $\beta$ can be 1 or 2 ). With overlap, for $\beta=1$, $\sup |\rho| \approx 1-\mathcal{O}\left(\Delta x^{1 / 8}\right)$, while for $\beta=2$, sup $|\rho| \approx 1-\mathcal{O}\left(\Delta x^{1 / 5}\right)$. Without overlap, in both cases, sup $|\rho| \approx 1-\mathcal{O}\left(\Delta t^{1 / 8}\right)$. Thus, if $\Delta t \approx \Delta x$, the performances with or without overlap are comparable, if $\Delta t \approx \Delta x^{2}$, the performances are higher with overlap.

\section{Numerical results}

In order to check the relevance of the theoretical best approximation, we run the case $\nu=0.2, a=1, c=0, \Omega=(0,6), T=2.5 . u(x, 0)=e^{-3(1.2-x)^{2}}$. The boudary conditions are $u(0, t)=0$ and $u(6, t)=0$. We chose $\Omega_{1}=(0,3.04)$, $\Omega_{2}=(2.96,6)$, which means $L=0.08$. The scheme is upwind in space, backward Euler in time, with $\Delta x=0.02, \Delta t=0.005$. The initial guess is random. Figure 3 shows that the theoretical best value of $p$ and $q$, coefficients of $P$ (represented by the star), is very close to the one numerically observed. 


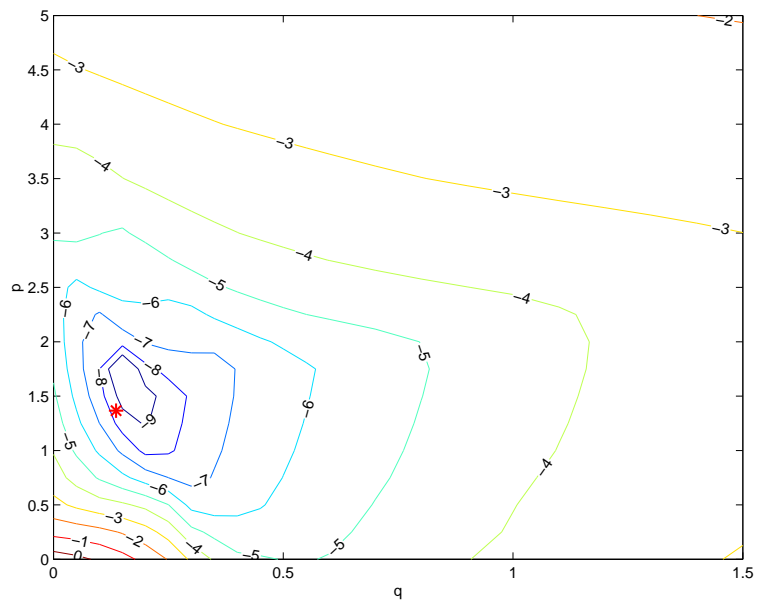

Fig. 3. Error after 5 iterations as a function of $p$ and $q$.

\section{Conclusion}

We have proposed a complete theory of a best approximation problem arising in sponge layers or SWR algorithms for parabolic equations. In one dimension it can be solved explicitely, thus providing the best answers to our questions. It remains to extend it in three directions: to rational fractions, to higher order, and to higher dimensions.

\section{References}

1. A. Bayliss and E. Turkel. Radiation boundary conditions for wave-like equations. Comm. Pure and Appl. Math., 33(6):707-725, 1980.

2. J. P. Bérenger. Three-dimensional perfectly matched layer for the absorption of electromagnetic waves. Journal of Computational Physics, 114:185-200, 1994.

3. D.Bennequin, M. Gander, and L. Halpern. Optimized Schwarz waveform relaxation for convection reaction diffusion problems. Technical Report 2004-24, LAGA, Université Paris 13, 2004. http://www-math.math.univparis13.fr/prepub/pp2004/pp2004-24.html.

4. B. Engquist and A. Majda. Radiation boundary conditions for acoustic and elastic calculations. Comm. Pure Appl. Math., 32:313-357, 1979.

5. M. Gander and L. Halpern. Absorbing boundary conditions for the wave equation and parallel computing. Math. of Comp., 74, 2005.

6. L. Halpern. Artificial boundary conditions for the linear advection diffusion equation. Math. of Comp., 46, Sept 1986.

7. M. Israeli and S. Orszag. Approximation of radiation boundary conditions. Journal of Computational Physics, 41:115-135, 1981.

8. V. Martin. An optimized Schwarz waveform relaxation method for unsteady convection diffusion equation. Applied Num. Math., 52(4):401-428, 2005. 